\title{
Dissolution Technologies: Update on New Features and Activities
}

Vivian A. Gray

Dissolution Technologies, Hockessin, DE USA e-mail:vagray@rcn.com n the August 2014 issue of Dissolution Technologies (1), which celebrated our twenty-year anniversary, was an article that commented on past achievements and gave some indication of future activies. In this article, we will update you on new features that will be of interest to the readers, authors, and advertisers.

We are very excited about the recently updated website that was redesigned to be mobile friendly. On the main page, current articles, calendar events, press releases, and books for online sale are more prominently displayed. Older press releases are easily accessible, as they are kept alive even as new content is posted. In addition, the online search feature for finding back issues remains intact.

Dissolution Technologies has been an Open Access journal from its inception in 1994; this means that articles are posted with free access on the website. No passwords, fees, subscriptions, or memberships are needed to access the articles. And very importantly, authors do not pay a fee for this open access. Most, if not all, "open" journals charge a fee up to thousands of dollars, yet an author's funding organization can demand that publications be published as open and freely accessible. Our articles are peer-reviewed and indexed. We would like to encourage those who need to publish in an open-access setting to consider Dissolution Technologies for their peer-reviewed publication needs on dissolution-related topics.

Digital Object Identifiers (DOI) provide permanent URL links to the articles and are assigned to every article. The DOI URL linking of the articles was activated in August 2014; however, all the technical articles have now been assigned DOls retrospectively. This means that if you published from 1994 onward, your article now has a DOI. Check it out.
We continue to strive to make the journal timely with Special Editions on pertinent topics. The next Special Edition, to be published February 2017, is on veterinary product dissolution. We are also inviting our experts to write review articles on various dissolution topics such as Surfactants, Performance Verification Testing (PVT), Gelatin Cross-linking, Buffers, Aerosols, Apparatus 3 and 4, Modeling, Hydrodynamics, $f_{2}$, Semisolids, and Critical Quality Attributes. If you have expertise in a particular dissolution-related topic and would like to contribute a manuscript, please contact the Research Editor at vagray@rcn.com.

The print edition format has been updated with crisp banners and formatting. With a change in layout and printers, the mailing and posting of the issues occur earlier than in past times.

Dissolution Technologies is tracked with Google Analytics. The suite of Google Analytics tools is largely graphical making it easy to answer questions of accessibility such as the most viewed/downloaded articles, where site visitors are located in the world, what people are searching for on the site, how users connect to the site, and much more.

This journal's purpose is to serve the community of dissolution scientists. The journal continues to improve in content, appearance, and accessibility. Any suggestions for improvements that our readers have would be most welcome.

\section{REFERENCES}

1. Gray, V. A. Historical Narrative on Dissolution Technologies, the Journal. Dissolution Technol. 2014, 21 (3), 41-42. DOI: 10.14227/ DT210314P41. 